\title{
TIPOLOGIE DI BAMBINI MASCHI MOSTRI NELL'IMMAGINARIO MEDIEVALE
}

\author{
Types of Monstrous Male Children in the Medieval Imaginary
}

Angela Giallongo
Università di Urbino
giallongoa@libero.it

Fecha de recepción: 20-III-2013

Fecha de aceptación: 27-III-2013

Astratto: Il contributo rileva l'importanza educativa dei bambini mostruosi in alcuni esempi significativi della letteratura medievale francese ed italiana. L'individuazione di quattro bambini maschi problematici è interpretata alla luce delle relazioni emotive con gli adulti.

Parole chiave: immaginario medievale, emozioni, bambini mostri maschi.

AвSTRACT: This essay investigates the important educational functions of monstrous children in some significant examples of Middle French and Italian literature. The focus on literary representations of four male children is inscribed in and linked to the emotional standards of adults' behaviour.

Keywords: medieval imaginary, emotions, monstrous male children.

Una via promettente per studiare la concettualizzazione medievale dell'infanzia consiste nel guardare più attentamente ai bambini mostri così come sono stati immaginati e al ruolo simbolico da loro esercitato nelle pratiche correnti del tempo. Ci sono infatti buone ragioni per pensare che rendere più esplicite tali immagini possa aiutare ad affrontare almeno alcune delle tante difficoltà della storia dell'infanzia, soprattutto in età pre-moderna. Esaminare più da vicino come fossero rappresentati i bambini mostruosi offre anche l'opportunità di analizzare la varietà di atteggiamenti emotivi provati dagli 
adulti nei loro confronti attraverso alcuni esempi significativi, forniti dalla letteratura francese ed italiana dei secoli XIII e XV. E' importante rilevare come allo stato attuale esista una fiorente letteratura storica sulla funzione dei mostri nella società medievale ${ }^{1} \mathrm{e}$ che soltanto da poco tempo la ricerca si sta occupando del tema qui in oggetto. Il recentissimo coinvolgimento degli storici per l'uso medievale dei bambini mostri ${ }^{2}$, spesso inverosimili e tuttavia agli occhi dei loro contemporanei misteriosamente convincenti, potrebbe svelare attraverso quali esemplari, reali e soprattutto immaginari, venissero divulgate e messe in scena le gerarchie naturali, le differenze generazionali, i valori educativi, le spinte emotive e le fobie sociali.

In un modo o nell'altro, l'attuale stato della ricerca invita a una valutazione critica circa la funzione dei bambini mostri e delle credenze sulle quali si era basata la loro invenzione. Inoltre, come ho già sottolineato nel Bambino medievale ${ }^{3}$, non ci si può sbarazzare del ruolo dell'immaginario nell'interpretazione delle concezioni, dei comportamenti collettivi e delle tradizioni educative del secondo millennio verso i minori. In questa sede -come si è detto-interessa principalmente l'idea di concentrare l'attenzione su quattro rappresentativi personaggi di sesso maschile chiamati a sostenere attivamente il modus educandi del periodo.

Gli inquietanti soggetti esaminati condividono infatti un eccezionale denominatore comune: non sono legati alla denigrazione del femminile, che aveva caratterizzato la mentalità del periodo fino a rendere l'intero gruppo delle figlie di Eva particolarmente popolare nel mondo teratologico. Questi sono infatti gli unici casi nei quali non entra direttamente in gioco lo stretto legame tra i mostri e le donne nel pessimo scenario, costruito ad hoc dal secondo millennio.

Del resto, la cultura medievale, adattandosi alla definizione classica di monstrum come prodigium, l'aveva intesa in conformità alle garanzie previste dal cristianesimo. I mostri pur non essendo strettamente vincolati al principio aristotelico, che li aveva

1 Fra gli studi fondamentali rimando a: C. Kappler, Demoni, mostri e meraviglie alla fine del Medioevo, tr. it.,Firenze, Sansoni, 1983; a M. H. Huet che, in Monstrous Imagination (Harvard University Press, 1993), ha mostrato come diverse opinioni filosofiche e scientifiche avessero attribuito fin dai secoli medievali al potere dell'immaginazione femminile la responsabilità dei processi procreativi anomali, successivamente attribuiti dal Romanticismo all'immaginazione maschile; $\mathrm{a}$ D. A. Williams che in Deformed Discours: The Function of the Monster in Mediaeval Thought and Literature (Montreal, McGill-Queen's University Press, 1996 Kingston) ha esaminato l'epistemologia medievale del mostro nel quadro filosofico e teologico insieme alle relative funzioni simboliche in campo letterario e artistico; infine, a C. Lecouteux, Les Monstres dans la Pensée médiévale européenne:Essai de Présentation (Cultures et Civilisation Médiévales, X).

2 Si veda il primo International Medieval Congress su You're So Juvenile: Monstrous Children in Medieval Culture, Western Michigan University, May 10-13, 2012; A. Czamowus, Inscription on the body: monstrous children in Middle English literature, Historia literatur obcych, Wydawnictwo Uniwersytetu Ślackiego 2009.

3 A. Giallongo, Il bambino medievale, Dedalo, Bari, 1990 (II ed. 1997), p. 77. 
considerati prodotti geneticamente carenti, erano soprattutto coltivati come messaggi inviati da Dio all'umanità, erano quindi sufficientemente fondamentali per aggregarsi a particolari insegnamenti didattico-morali e per essere interpretati in chiave allegorica. Questo mutamento radicale di mentalità, constatato da C. Klapper, si rispecchia nella visione agostiniana: i mostri appartenevano all'armonia dell'universo, come tutte le altre cose del mondo, erano il segno del potere divino sulla natura ${ }^{4}$.

\section{FIGLI DI GIUDA}

Nel XIII secolo si diffuse l'idea dei bambini fatali ${ }^{5}$, ben individuati dallo storico italiano della letteratura A. Graf in Miti, leggende e superstizioni del Medioevo (1892-93). Sono stati infatti i suoi lucidi, minuziosi studi a sottolineare la frequenza di questo tema e a suggerire un aspetto molto importante ai nostri occhi per la storia delle emozioni e delle mentalità del periodo preso in esame.

Non è quindi privo d'interesse richiamare brevemente il ruolo di questi protagonisti in qualche modo terrificanti e in ogni caso capaci di alimentare il discorso letterario, astrologico, religioso, educativo e morale. Di questi malnati, che a detta di tutti destavano orrore, si occupava un gruppo di esperti: astrologi, indovini, personaggi umani, sovrannaturali. Ma entravano in gioco anche eventi ordinari e straordinari (semplici sogni o animali parlanti) in grado di leggere il futuro. In prossimità di un parto, gli specialisti erano autorizzati a predire la morte e/o gravi sciagure individuali e collettive. I genitori e i tutori accorti, come testimoniano numerosi racconti, per evitare e annientare l'imminente flagello chiudevano i predestinati e le predestinate in un castello o in una torre o più semplicemente li abbandonavano nella foresta, in una zona deserta, o li gettavano in mare aperto.

La credenza medievale nel fato veniva inoltre confortata dal mito di Edipo, diventato involontariamente precursore della leggenda di Giuda. Il mondo letterario italiano fantasticò molto sull'apostolo traditore, sulla sua vita scellerata e sull'innegabile fatto che era destinato a causare danni fin dal primo vagito. Ma in genere, secondo Graf, le favole sugli scellerati mostravano anche l'occulto destino che li aveva spinti a compiere fin dalle fasce misfatti contro la loro volontà e che soprattutto il carattere insidioso di questa particolare mostruosità si manifestava solamente dopo il compimento dei crimini ${ }^{6}$. Verso la fine del XIII secolo, Jacopo da Varagine (1228 ca. - 1298) collocava, nella sua popolare Legenda aurea, Giuda come primo di tutti i malnati'. A Gerusalemme, Ciborea, sua

Cfr. C. Kappler, op. cit., p. 8 e p.183.

A. Graf, Miti, leggende e superstizioni del Medioevo, Mondadori, Milano, 1996, p. 199.

Ivi, p. 189.

Giuda Iscariota in Jacopo da Varagine, Legenda aurea, Einaudi, Torino, 2007, p. 424. 
madre, non a caso aveva sognato di mettere al mondo uno rampollo terribile che avrebbe rovinato il popolo ebraico e che pertanto l'unica soluzione era quella di metterlo, come Mosè, in una cesta da destinare alle onde del mare. Inutile raccontare che Giuda verrà salvato, che si macchierà di numerosi crimini, compreso il fratricidio, il parricidio, l'incesto e che andrà, com'è noto, fino in fondo, sia con il «tradimento del suo maestro» sia con il suicidio. Un atto che veniva condannato non soltanto sul piano etico, religioso ma anche su quello giuridico: il suicida, agli occhi del medioevo, era contemporaneamente un peccatore, un indemoniato e un criminale.

La biografia di Jacopo da Varagine sull'infanzia di Giuda obbedisce comunque a uno schema preciso: premonizione, abbandono fin dalla nascita e denuncia dei continui maltrattamenti inflitti al fratellino putativo, ucciso alla prima occasione: con questo marchio d'eterna infamia Giuda concludeva, sulle orme di Caino con Abele, la sua triste infanzia ${ }^{8}$. Ecco l'essenziale di questo testo fondamentale sul malnato.

Prescindendo da questa biografia e da altre simili, basate sul parricidio, sul matricidio, sull'incesto e su altri delitti, risulta evidente la teoria secondo la quale tutti questi comportamenti aberranti erano voluti da un fatum invictum, una specie di forza occulta alla quale nessuno poteva sfuggire. Neanche i Santi. Come mostrano le diverse leggende ripescate da Graf anche nella tradizione latina (per esempio nelle varie redazioni dei Libri dei Sette Savi). Comunque, non sempre i fanciulli fatali dei racconti religiosi e profani diventavano dissoluti peccatori, talvolta acquistavano da adulti in tutte le classi sociali e presso la comunità grande dignità.

Ma, d'altra parte, proprio su questo punto, individuato da Graf, si può riflettere sul fatto che i sottesi discorsi educativi erano spesso specchi ingannevoli, perché peccavano di ottimismo, quando attraverso i loro exempla insegnavano che non vi era nessun animo così mostruoso da non poter essere riscattato dalla virtù della penitenza e della santità. Forse la cosa era più facile da dirsi che da farsi. Era infatti più semplice per gli adulti rifiutarsi di allevare figli che avrebbero minacciato la loro stessa vita, insieme a quella del cielo e della terra. E con la storia di Giuda nella Legenda aurea si affacciava in modo convincente il «mitema della vittoria sul mostro» ${ }^{9}$ con la certezza che fosse irrimediabilmente tale fin dalla nascita.

\section{PULICANE}

Nel XIV secolo cominciarono a circolare in area italiana diversi racconti animati dal personaggio di Pulicane, un essere umano con testa canide, dotato ovviamente

\footnotetext{
Ivi, p. 425.

9 P. E. Dauzat, Giuda. Dal vangelo all'Olocausto, tr. it., Arkeios, Roma, 2008, p. 66.
} 
di un'infallibile fiuto. A renderlo noto era stato uno scrittore e cantastorie fiorentino, Andrea da Barberino (1370-1431) con il suo principale lavoro I reali di Francia, ristampato con successo fino al XIX secolo. Qui in un ampio ventaglio di scenari, che raccontavano, in prosa vernacolare, le complesse vicende e avventure della dinastia carolingia, ci si imbatte in un discendente dell'immaginario religioso egiziano, asiatico e del folkore russo. Per il suo aspetto Pulicane non poteva essere considerato un cristiano, veniva pertanto inevitabilmente incluso tra le grandi fobie dell'immaginario collettivo, dove era designato come capro espiatorio. La morte sul rogo che lo attende al primo vagito riassume perfettamente l'atmosfera emotiva dell'epoca. Essendo frutto "d'una donna e d'uno grande mastino», quindi un «mezzo cane», il neonato era già, come racconta Andrea da Barberino, stato condannato dal re Erminione alle fiamme ${ }^{10}$.

Ma la nostra fonte proietta una luce benevola sul modo di vedere quest' infanzia così imperfetta. Come testimoniano le parole rivolte all'interessato dalla protagonista Drusiana, la figlia del re che a nove anni prese l'iniziativa di proteggere il neonato imputato, già predestinato alle fiamme:

"O Pulicane, è questo il merito che tu mi rendi del servigio che io ti feci, quando io ero d'etá di nove anni, che tu fusti menato per essere arso nel fuoco ardente, e dicevano che tu eri nato di mortale peccato, e generato d'animale inrazionale, come era uno mastino, in una femina razionale, e io ti domandai di grazia al padre mio, e scampasti dalla morte?» ${ }^{11}$.

Il caso eccezionale rivela attraverso la sensibilità di Drusiana le reazioni degli adulti combattuti tra l'esigenza di reprimere violentemente il frutto del peccato e la curiosità di «allevare e nodrire» un bambino mostro. A Pulicane, che era comunque cresciuto come un cane addomesticato legato ad una catena, perché era "troppo robusto», verrà infatti promesso, nell'intreccio delle vicende delle quali era protagonista, non soltanto il battesimo e la libertà ma anche l'opportunità di affermarsi socialmente come «un gran signore» e di essere infine tenuto " molto caro a corte» ${ }^{12}$.

Le lacrime di commozione dell'ibrido attirano, in un'altra scena, su di lui e sulla sua storia una sorta di approvazione collettiva. Ma la sopravvivenza di atteggiamenti tradizionali si manifesta ugualmente con la morte del protagonista che finisce i suoi giorni onorevolmente prima di beneficiare dei sacramenti religiosi, degli onori sociali e dei promessi legami affettivi.

$\overline{10}$ Andrea da Barberino, I reali di Francia, a cura di G. Vandelli e G. Gambarin, Bari, Laterza, 1947, Libro IV, Capitolo XXVII. p. 326.

11 Ivi, Libro IV, Capitolo XXIX, p. 347.

12 Ivi, Libro IV, Capitolo XXVII, p. 325. 
Nonostante la fragilità di questa testimonianza si può ricavare una certa considerazione, a partire dal XIV secolo, per la non colpevolezza del bambino, ritenuto frutto di rapporti sessuali anomali: comunque, non voluti, secondo le credenze del tempo, dalla madre ma dal particolare interesse sessuale nutrito dai Cinocefali per le donne civilizzate o, come si diceva allora, «razionali». E' possibile inoltre intravedere uno slancio embrionale di tolleranza e/o di ricettività verso le imperfezioni fisiche.

In base all'ampia documentazione fornita da D. Gordon White ${ }^{13}$ sui miti, sulle leggende e sulle credenze dei Cinocefali in Europa, Asia e India, si evince che la particolare attrazione medievale per gli questi ibridi era stata soprattutto alimentata da varie tradizioni orientali (gnostica, copta e nestoriana) che erano state integrate dalle agiografie, dalle enciclopedie ${ }^{14}$ e dalle opere letterarie occidentali. Inoltre, grazie ad Agostino, i Cinocefali, insieme ad altre razze fantastiche, essendo visti come parte integrante dell'economia della salvezza, venivano ampiamente allegorizzati e moralizzati. Il successo del santo Cristoforo Cinocefalo negli affreschi e nelle icone delle chiese bizantine è la sorprendente testimonianza dell'immagine ideale dell'ibrido cristianizzato. Nella Passio Sancti Christophori martyris, presente in vari testi patristici e che ebbe molta diffusione in epoca medioevale, veniva narrata la leggenda del Santo, un soldato Cinocefalo convertitosi appunto al Cristianesimo. White ci mostra anche un autore altomedievale del IX secolo, il monaco benedettino Ratramno di Corbie (Ratramnus) che nella Epistola de Cynocephalis incoraggiava a considerare i Cinocefali esseri umani ${ }^{15}$.

Ho insistito su questo aspetto perché potrebbe aver avuto un ruolo costruttivo nello sviluppo delle sensibilità collettive verso le nascite cosiddette mostruose. Da una parte appare infatti moralmente appropriato nel racconto di Andrea da Barberino l'insolito salvataggio di un neonato non conforme alla norma; questo significa che grazie a questa scelta -esaltata come una "cosa meravigliosa»- Pulicane aveva avuto l'occasione di crescere correndo più forte di un daino e di un cervo, di mettere alla prova il suo «buon naso» e di diventare anche un ottimo arciere ${ }^{16}$.

13 Cfr. D. Gordon White, Myths of the Dog-Man, University Of Chicago Press, 1991.

14 Nelle enciclopedie del XIII secolo i Cinocefali venivano inaspettatemente considerati un specie umana, dotati di un'anima razionale e quindi discendenti di Adamo: cfr. Ph. Franceschetti, Mission et intégration sociale: le cas des cynocéphales au IXe siècle in Vivre en société au Moyen Age. Occident chrétien, VIe-XVe siècle cur. C. Carozzi - D. Le Blévec - H. Taviani-Carozzi, Aix-en-Provence 2008 pp. 177-179; E. A. Matter The Soul of the Dog-Man: Ratramnus of Corbie Between Theology and Philosophy in Filosofie e teologie nel medioevo, (a cura di) M. T. Fumagalli Beonio Brocchieri M. Parodi in «Rivista di storia della filosofia» 61, 2006, pp. 43-53.

15 D. Gordon White, Myths of the Dog-Man, cit., p. 18.

16 Andrea da Barberino, I reali di Francia, cit., Libro IV, Capitolo XXVII, p. 327. 
Dall'altra, era poi apparso con lo stesso esprit de finesse il fenomeno di un cane che era diventato santo e salvatore di bambini ${ }^{17}$. Il libro di Schmitt ha localizzato la leggenda nel nord della Francia con i culti del mondo rurale del XIII secolo: qui i bambini ammalati venivano portati al Saint Guinefort (un levriero che aveva salvato la vita a un lattante ma che era stato martirizzato per errore dal suo padrone) nella speranza di aiutarli. Un manoscritto del XIII secolo di un inquisitore aveva documentato la distruzione da parte delle autorità ecclesiastiche di un rito popolare che era sopravvissuto fino al XX secolo. Indipendentemente dai conflitti spirituali tra le credenze rurali e la chiesa, rimane il fatto che lo studio di Schmitt ha messo bene in luce la predominante presenza femminile in questi rituali. Nel paradosso di un cane santificato si può quindi intravedere il bisogno intimo delle madri di trovare in buona fede sia un rimedio alle infermità dei figli -abbandonati temporaneamente nelle mani di colui che avrebbe deciso della loro sorte- sia un supporto di fronte al rischio della loro perdita. Desideravano, dedicandoli al santo levriero, migliorare la salute dei loro piccoli ammalati o trovare un conforto al dramma della irrimediabile separazione.

Ma alla luce di una favola dei fratelli Grimm, si può pensare che non era raro il caso in cui si giustificava l'infanticidio di fronte una condizione patologica estrema: il neonato era stato infatti gettato in un fiume, perché era del tutto simile ad un cane ${ }^{18}$.

\section{PUER DIABOLICUS}

La storia di Robert le diable ${ }^{19}$, composta da un anonimo agli inizi del XIII secolo, documenta l'infanzia esemplarmente negativa di un essere sprofondato fin dalla culla nel male. La leggenda costituisce un osservatorio privilegiato sulle preoccupazioni e sulle fantasie sociali di un'epoca, sostanzialmente convinta, in questo caso, che i genitori senza figli potessero rivolgersi direttamente al diavolo.

La madre di Roberto infatti dopo interminabili preghiere e una vana attesa nei diciassette anni di matrimonio, si era infine raccomandata al maligno per concepire con il marito quel figlio così a lungo desiderato. A tempo dovuto, la duchessa di Normandia partorì, senza buttarsi di fatto in braccio a Satana. Ma l'infante, che non era tecnicamente un ibrido, in quanto frutto di un patto più contrattuale che biologico, rivelò subito i peggiori istinti: mise a dura prova la pazienza e l'incolumità delle nutrici con calci e morsi feroci al seno. Queste azioni venivano collegate alla rabbia ed erano categorizzate nei termini ristretti del comportamento maligno più che primitivo. Non

17 Cfr. J. C. Schmitt, Le Saint lévrier Guinefort, guérisseur d'enfants depuis le XIIIe siècle, Paris, Flammarion, 1979.

18 J. e W. Grimm, I tre uccellini in le Fiabe del focolare, Mondadori, Milano, 1970, p. 337.

19 Si veda l'edizione curata da É. Gaucher, Robert le Diable, Champion, Paris, 2006. 
è quindi una sorpresa riscontrare nelle donne una grande apprensione: esse cercarono comunque di limitare il danno, allattando questo poppante, particolarmente turbato ed eccitato, con un corno usato come biberon. Roberto quindi crebbe "grosso e bello" e con l'ostinata convinzione che i suoi morsi come altre offese corporali dimostravano la sua invincibile forza. Era infatti cattivo, prepotente, crudele, orgoglioso, rabbioso e pigro come il padre spirituale. Ben quattro maestri non riuscirono a fargli intendere la ragione. Era nemico dei santi, fracassava gli oggetti di chiesa e, prima ancora di aver compiuto quattordici anni, aveva già spedito all'altro mondo diversi preti. Inoltre, si rinvigoriva a vista d'occhio: a un anno ne dimostrava sette e a venti era il più alto e il più forte dei suoi coetanei.

Descrizioni e osservazioni analoghe si trovano nelle numerose, complesse versioni orali e scritte sulla vita di Merlino. Il personaggio era stato genialmente costruito dall'ecclesiastico scozzese Geoffroy of Monmouth nel XII secolo per salvare la reputazione di un principessa reale, una monaca che aveva avuto un figlio illegittimo. Nella sua Historia regum Britanniae ${ }^{20}$ (1148-1155) Merlino era stato infatti proposto come un essere generato da un spirito diabolico che aveva avuto un rapporto sessuale con una un vergine addormentata. Era quindi un caso diverso da quello di Robert le diable. Inoltre, un tempestivo battesimo aveva di fatto impedito al maligno di esercitare i suoi poteri negativi attraverso il figlio. Ma in una illustrazione di un manoscritto Ystoire de Merlin del XV secolo il neonato viene ritratto, in base alla teoria aristotelica, peloso e nero come il padre. Come Roberto accelerava i tempi della sviluppo: a dodici mesi mostrava più di due anni, mordeva a pieni denti invece di poppare e rideva dei pianti di sua madre. Ma soprattutto parlava: «Nel sentirlo, le donne si spaventarono e commentarono: «Non è un bambino ma un diavolo che capisce ciò che diciamo» ${ }^{21}$.

Va precisato che secondo le credenze popolari nordiche erano i changelins -i figli dei demoni che si sostituivano nella culla ai neonati umani- ad avere questo potere. Non a caso, per verificare l'accaduto, gli adulti, come ci hanno mostrato diverse favole dei fratelli Grimm, usavano compiere davanti al sospettato una serie di azioni assurde in modo da indurlo a manifestare la sua natura non umana con frasi del tipo: "ho vissuto cinquecento anni e non ho mai visto niente di simile».

La convinzione che la vera età di questi esseri fosse superiore a quella mostrata suggerisce indirettamente come il bambino percepito con facoltà superiori a quelle rico-

20 Goffredo di Monmouth, Storia dei re di Britannia (traduzione dal latino), Introduzione e note di G. Agrati e M. L. Magini; Presentazione di F. Cardini, ed. U. Guanda, Milano, 2005.

${ }^{21}$ Ystoire de Merlin (XV secolo) citato da C. Gaignebet- J.D. Lajoux. Arte profana e religione popolare nel Medio evo, tr. it., Fabbri, Milano, 1986, p. 307. 
nosciute mediamente appropriate all'età, venisse considerato dal XII secolo un evento non naturale, straordinario, divino o semplicemente diabolico.

Nonostante l'interesse medievale per l'infanzia di Merlino, è curioso il fatto che la tradizione letteraria europea preferì, in modo particolarmente evidente dal XIX secolo, rappresentare il più leggendario protagonista del ciclo arturiano con l'aspetto di un vecchio stregone che non era mai stato bambino. In base al quadro delineato da A. Berthelot sul ruolo ambiguo di questo personaggio si evince che erano già stati i testi in lingua vernacolare ad introdurre la tendenza, nel tardo XVI secolo, a descrivere Merlino come un adulto preferibilmente vecchio ${ }^{22}$ e a non trovare più attraenti le sue avventure infantili. Diversamente il pubblico medievale assecondava questo bambino problematico che parlava precocemente e diceva cose molto intelligenti. Un comportamento che in ogni caso era considerato un privilegio dell'età matura. Il puer senex, la cui esistenza era fondata sull'imprevedibile fusione dei contrari, aveva infatti il raro dono di parlare, di dire verità, di convincere e di persuadere gli adulti. I lettori e dagli ascoltatori erano affascinati sia da una figura dalle sembianze infantili che aveva la saggezza di un vecchio sia dall'idea della coincidentia oppositorum, basata appunto sulla presunzione della inevitabile corrispondenza tra prima infanzia ed estrema vecchiaia. Nonostante il successivo cambiamento di gusti a riguardo, gli adulti del tempo erano talmente desiderosi di credere in questa formula da aver preventivamente accettato il principio secondo il quale Merlino non aveva età: poteva attraversare contemporaneamente, per rispetto al principio del rovesciamento parodico dell'esperienza reale, i sette e i settanta anni.

Forse sensibilizzati delle nascenti opinioni mediche sull'importanza del gioco nelle prime età ${ }^{23}$, diversi adulti d'entrambi i sessi degli ambienti colti si distraevano di fronte alla messa in scena di un bambino che si trastullava anche con il tempo.

22 A. Bertrhelot, Merlin Puer senex par excellence in A. Classen, Old Age in the Middle Ages and the Renaissance. Interdisciplinary Approach to a Neglected Topic, Walter de Gruyter, Berlin 2007, pp. 251-262.

23 La scoperta dell'importanza del gioco è mirabilmente visibile in Aldobrandino da Siena, autore nel 1256 del Régime du corps: un trattato scritto in lingua provenzale che era stato dedicato alla contessa Beatrice di Provenza, suocera del re di Francia e madre di ben quattro regine. Grazie a questa fortunata circostanza il manoscritto circolò, fra il XIII e il XIV secolo con successo nelle case regnanti inglese, ungherese, tedesca e siciliana. In Italia venne inoltre volgarizzato dal medico-notaio Zucchero Bencivenni nel 1310 sotto il nome la Sanità del corpo e messo a disposizione di un pubblico ancora più vasto rispetto a quello previsto dall'originale. Va infine detto che in questa opera venivano riassunte, elaborate e illustrate le idee della tradizione medica del periodo: particolarmente interessante risulta pertanto la sensibilità mostrata da Aldobrandino per il gioco che era stato collocato fra le attività principali che garantivano non soltanto la salute del corpo e dell'anima ma il rispetto per la naturale vivacità dell'infanzia: lo stato d'animo dell'infanzia era infatti attratto da "tutte cose di giocho et di sollazzo». Aldobrandino da Siena, La sanità del corpo volgarizzato da Zucchero Bencivenni, Cap. XVII, C 17 t, Cod. II-ii-85 e Cap. XVIII, C.17; C,17 t,. Biblioteca Nazionale di Firenze. 
Ma se riflettevano più attentamente sul puer senex potevano ricredersi e considerarlo un mostro. Appena nato, le donne di fronte ai quei tratti fisici bestiali, non umani, selvaggi, diabolici ${ }^{24}$ ebbero infatti paura ${ }^{25}$.

\section{PUER FERUS}

La lunga, oscura e affascinante storia dei bambini sopravissuti per auto sostentamento o allevati da specie animali suggestionava i gusti letterari ed artistici: dalle foreste, luoghi oscuri ed inquietanti appariva l'aspetto informe, sporco, muto del bambino selvaggio. La sua presenza era già stata alimentata dalle leggende greco-latine, ma non è da escludere che il ritrovamento di esseri vissuti nella foresta, abbia ispirato questo tema nei romanzi fiamminghi, carolingi e ugro-bizantini. L'iconografia popolare aveva a sua volta identificato questo personaggio con il culto dell'orso, celebrato nelle feste pagane, cristiane e celtiche ${ }^{26}$. Ad Ambierle, in una chiesa del XIV secolo, troneggia in uno stallo una coppia di selvaggi con figlioletto: tutti e tre coperti di un abbondante pelame arruffato.

La chanson du chevalier au cygne restituisce uno dei primi ritratti attendibili del bambino selvaggio: era villoso come un lupo o un orso incatenato; aveva le unghie grosse e i capelli impiastricciati; la testa cinghialesca e portava un bastone squadrato, era vestito di foglie, sotto tutto peloso: sembrava proprio un folle o un selvaggio ${ }^{27}$.

La descrizione rievoca quella fatta da Itard verso la fine del Settecento di fronte al dodicenne ritrovato nella selva dell'Aveyron.

Agli occhi dell'uomo medievale la foresta era la patria primitiva di chi non aveva leggi né un'organizzazione sociale e religiosa; era un luogo popolato dagli antropofagi, da esseri armati di clava e/o di coda (per l'epoca le scimmie facevano parte della stessa categoria) o da inoffensivi vegetariani, qualche volta scambiati per santi. La foresta era, in ogni caso, il luogo adatto ad individui incompetenti a livello gastronomico -visto che

24 E'stata l'immaginazione di Robert de Boiron a introdurre attraverso un'analisi dettagliata della nascita di Merlino il tema della dimensione diabolica:»Ils [le diable et les démons] tombent d'accord et conviennent d'engendrer un home capable de séduire l'humanité entière. [...] C'est ainsi que le diable entreprit de créer un être qui eût sa mémoire et son intelligence pour se jouer de Jésus-Christ». Robert de Boron, Merlin. Roman du XIIIe siècle. Présenté, traduit et annoté par A. Micha, Paris, Flammarion, 1994, p. 25.

25 Ystoire de Merlin (XV secolo) citata da in C. Gaignebet- J.D. Lajoux, op. cit., p. 307.

26 C. Gaignebet- J.D. Lajoux., op. cit., p. 90.

27 Godefroy de Bouillon La Chanson du Chevalier ou Cygne, éd. Hippeau, Paris, 1874 e il commento di C. Gaullier-Bougassas, «Le Chevalier au Cygne à la fin du Moyen Âge : renouvellements, en vers et en prose, de l'épopée romanesque des origines de Godefroy de Bouillon», La Fortune de l'épopée médiévale, études réunies par F. Suard, Cahiers de Recherches médiévales, 2005, pp. 165-199. 
mangiavano di tutto dalla carne cruda alle foglie- e completamente sprovveduti nell'arte dell'igiene e della parola.

Molte di queste caratteristiche si addicevano al bambino. Nel vederlo camminare a quattro zampe, nel crederlo estraneo alle leggi, alle facoltà razionali e ai sentimenti, nell'osservarlo portare alla bocca qualsiasi cosa, era inevitabile prestargli la parte del selvaggio da addomesticare e da civilizzare. Il filone letterario giustificava essenzialmente in due modi la presenza del bambino selvaggio: per nascita o per allattamento. Nel primo caso rientravano tutti quei personaggi che come Pulicane o Merlino avevano avuto per padre un animale, un gigante o il maligno e per madre una donna civilizzata. Infatti, è particolarmente palese in questi personaggi l'influenza della teoria aristotelica sul concepimento, che aveva favorevolmente spostato, in questa circostanza, il centro dell'attenzione sulla donna, la cui funzione ritenuta biologicamente passiva trionfava soltanto sul principio maschile negativo e bestiale ${ }^{28}$.

In un periodo profondamente dominato dall'idea dell'imperfezione femminile e dalla paura delle sue pericolose conseguenze, attraverso il contaminante flusso mestruale, sulla prole, queste erano le uniche occasioni che non attribuivano alla donna la responsabilità delle nascite anomale.

Nel secondo caso, valeva il principio che fosse l'allattamento da parte di un animale selvatico a determinare lo stato del bambino. Nella trama di una chanson de gestes del XIV secolo ${ }^{29}$ e di un romanzo francese ${ }^{30}$ del XV secolo, Valentino e Orsone, veniva fornita la seguente spiegazione magico-scientifica:

«Nutrito del latte dell'orsa, il piccolo divenne peloso come un animale selvaggio. Camminava per i boschi e crebbe in fretta e cominciò ad assalire le altre bestie della foresta, tanto che tutte ne avevano paura e fuggivano appena lo vedevano, dal momento che egli non ne temeva alcuna, per quanto feroce, e,conducendo una vita da fiera non aveva timore di nulla nello stato in cui era. Nel giro di quindici anni il fanciullo era diventato forte, grosso e possente e nessuno osava attraversare più la foresta. Abbatteva e uccideva bestie e uomini e,come le bestie, ne mangiava la carne cruda; conduceva insomma una vita più ferina che umana. Essendo stato allevato da un orso ed essendo coperto di pelo come un orso, fu chiamato Orsone...Faceva vita da animale selvaggio, senza abiti per coprirsi e senza pronunciare una parola ${ }^{31}$.

\footnotetext{
28 Aristotele, Riproduzione degli animali. Parti degli animali, II, 737 a.

29 Sul commento a questa leggenda si veda: A. Dickson, Valentine and Orson: a study in late medieval romance, New York, Columbia University Press, 1929.

30 Con la storia di Valentin et Orson. Lyon, Jacques Maillet, 1489 molto popolare nel medioevo è nato ufficialmente il tema del bambino selvaggio.

31 Qui è stata utilizzata la versione italiana a stampa : G. Leggio, Istoria Dei Due Nobilissimi E Valorosi Fratelli Valentino E Orsone: Figliuoli Del Magno Imperatore Di Costantinopoli E Nipoti Del Re
} 
La ferma convinzione sul fatto che i bambini crescessero fisicamente era accom pagnata dalla seguente idea: al di fuori dell'ambiente umano i bambini potevano soltanto regredire. L'intreccio dei fratelli gemelli, separati dal destino, simboleggiava con la storia di Valentino e Orsone la contrapposizione tra natura e cultura. Il primo era stato felicemente allevato a corte, luogo ritrnuto ottimale dal XII secolo per favorire lo sviluppo umano; mentre il secondo, cresciuto nei boschi e allattato da un animale, era il necessario risvolto negativo della medaglia. Il caso dei gemelli semplificava le dinamiche sottese alla contrapposizione civiltà-barbarie. Il puer ferus, o meglio l'infanzia selvaggia dell'eroe, si inseriva perfettamente in questo schema profondamente dualistico dell'immaginario medievale.

\section{CONCLUSIONE}

La breve ricognizione su alcune tipologie medievali di bambini di sesso maschile outsiders ha suggerito in queste pagine l'importanza vitale di approfondire dal punto di vista della storia dell'infanzia il ruolo dell'immaginario nelle espressioni delle emozioni sociali e delle teorie educative. Pertanto, passando in rassegna quattro infanzie di figure popolari importanti -i bambini fatali, ibridi, diabolici e selvaggi- e concentrando l'attenzione sulla loro capacità di suscitare varie emozioni, sono state colte tracce di complesse tensioni adulte (paura, violenta aggressività, disperazione, in particolare delle madri e delle nutrici. mista ad ostilità, curiosità e senso della protezione) e consistenti indizi sui valori educativi, ritenuti più importanti dagli autori esaminati nel periodo compreso fra il XIII e il XV secolo.

Si è sottolineato inoltre che ciò che ha contraddistinto questi bambini mostri, che rimanevano comunque membri di una categoria degradata, é il fatto che le loro anomalie non erano imputabili alle madri, frequentemente implicate nell'accusa, soprattutto da parte degli autori dei trattati medici, di aver corrotto fisicamente la prole. Presi nel loro insieme, questi personaggi medievali contribuivano, con i loro stravaganti aspetti esteriori e con i loro comportanti turbolenti, a rafforzare il senso della distanza e dell'alterità nelle relazioni fra adulti e minori.

Diversamente infatti dalle aspettative agostiniane, che avevano comunque negato, nella Città di Dio, la presenza dei bambini in paradiso, le illustrazioni dei mostri nel Cinquecento -viste da C.Klapper come una continuazione della tradizione del secondo millennio- sembrano suggerire reazioni emotive piuttosto sommarie. In particolare, la miniatura dell'umanista tedesco Sebastian Brant (1457-1521). Nel «De puero quadrupede in agro fiorentino ex equa nato" si vede infatti un contadino decapitare in pieno giorno un fanciullo dal corpo equino. Il mostro con la testa da bambino, simbolo del

\footnotetext{
Pipino,. Edizione Adriano Salani, Firenze, 1920, p. 45.
} 
male nascente, doveva essere immediatamente eliminato, come recitava la didascalia con «horrore atque abominazione». Questa era dunque la reazione ritenuta moralmente pertinente.

La sorte migliore individuata con Pulicane, il bambino con la testa da mastino, pur esprimendo un atteggiamento negativo -il soggetto in questione in quanto tale doveva essere tenuto a distanza, alla catena- non provocava comunque un violento rifiuto nei suoi confronti. Da Andrea da Barberino sappiamo ormai bene che Pulicane si rivelò un eccellente combattente e che venne, grazie a un lampo di empatia femminile, salvato alla sua nascita, dal rogo. Molte sono quindi le conseguenze interessanti dovute a questa nuova tendenza, specialmente nel campo dell'infanticidio e dell'abbandono dei minori, nati deformi. La capacità di coltivare un legame nei loro confronti potrebbe aver gradualmente incoraggiato lo sviluppo di positive competenze sociali.

Ma per tornare più direttamente all'argomento principale, cioè alle preoccupazioni collettive sollevate dall'invenzione dei bambini fatali, mostri, diabolici e selvaggi, bisogna ricorrere alla presa di posizione assunta nel XII secolo da Chrètien de Troyes, nel Perceval, sui limiti dello stato di natura. Nell'indicare lo stato di natura, inferiore all'educazione, Chrètien aveva energicamente suggerito che chi veniva lasciato a se stesso o ai margini della comunità, non avrebbe sviluppato nessuna capacità umana ${ }^{32}$. E'quindi possibile individuare attraverso le storie di questi bambini problematici, occasionati dai romanzi del secondo millennio, le questioni emotivamente rilevanti per gli adulti medievali, ora coinvolti in azioni violente e aggressive ora disposti ad invocare il senso della protezione nei loro confronti ora affascinati dalla loro alterità. Negli ultimi due casi, gli adulti erano incoraggiati a vedere l'umanità dei presunti imputati. Ancora, si può supporre che il successo di questi personaggi abbia probabilmente contribuito a aprire ed educare il pubblico adulto femminile e maschile a una maggiore percettività e arricchimento nelle relazioni affettive familiari e sociali con i minori.

32 Chrètien de Troyes, Perceval, Mondadori, Milano,1983 p. 9. 